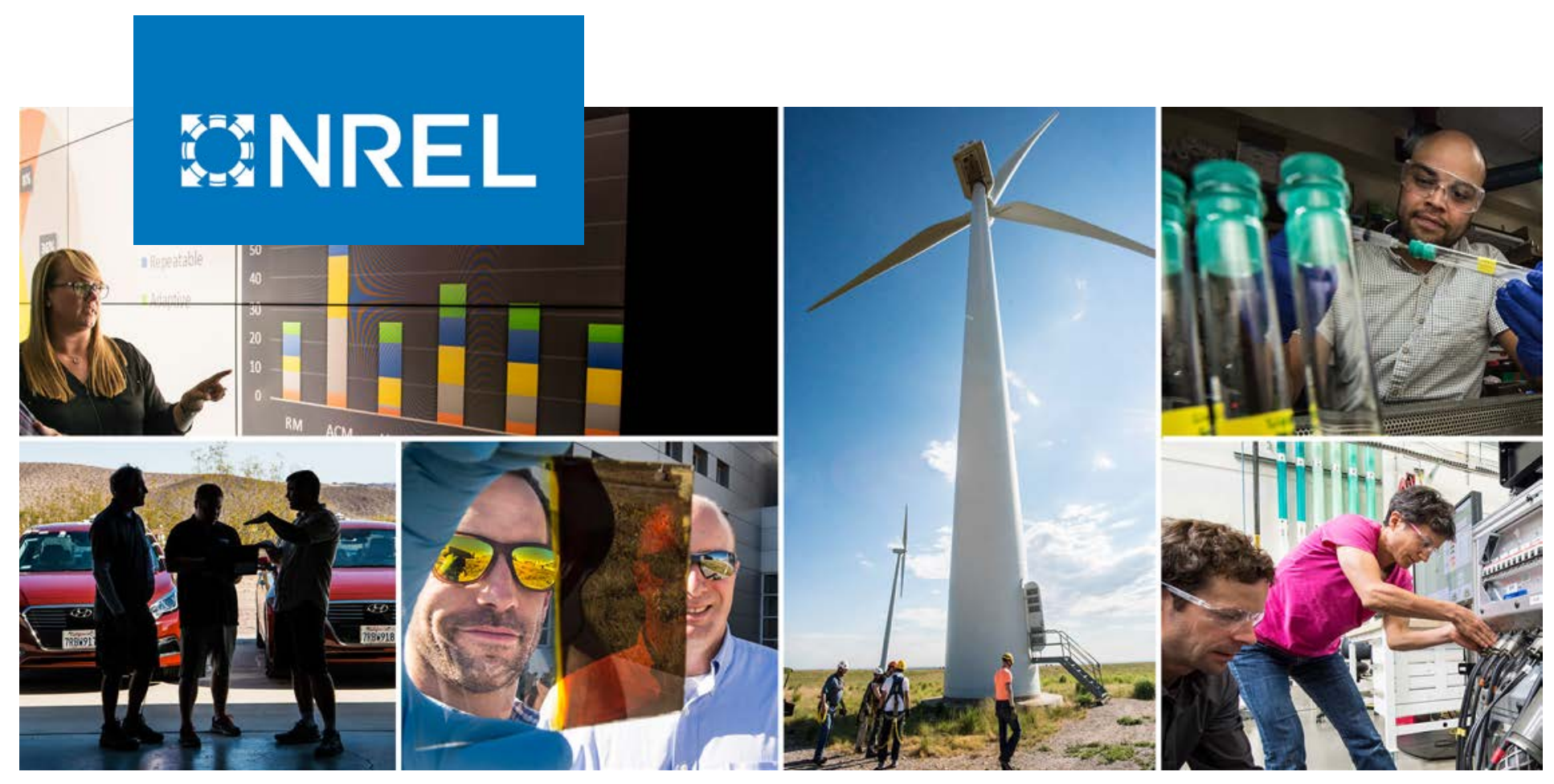

\title{
Integrating Smart Plug and Process Load Controls into Energy Management Information System Platforms: A Landscaping Study
}

Rois Langner and Kim Trenbath

National Renewable Energy Laboratory

NREL is a national laboratory of the U.S. Department of Energy Office of Energy Efficiency \& Renewable Energy

Operated by the Alliance for Sustainable Energy, LLC

This report is available at no cost from the National Renewable Energy Laboratory (NREL) at www.nrel.gov/publications.
Technical Report

NREL/TP-5500-74080

June 2019 


\section{EAREL}

Integrating Smart Plug and Process

Load Controls into Energy Management

Information System Platforms:

A Landscaping Study

Rois Langner and Kim Trenbath

National Renewable Energy Laboratory

\section{Suggested Citation}

Langner, Rois, and Kim Trenbath. 2019. Integrating Smart Plug and Process Load

Controls into Energy Management Information System Platforms: A Landscaping Study.

Golden, CO: National Renewable Energy Laboratory. NREL/TP-5500-74080.

https://www.nrel.gov/docs/fy19osti/74080.pdf.

NREL is a national laboratory of the U.S. Department of Energy Office of Energy Efficiency \& Renewable Energy Operated by the Alliance for Sustainable Energy, LLC

This report is available at no cost from the National Renewable Energy Laboratory (NREL) at www.nrel.gov/publications.

Contract No. DE-AC36-08GO28308
Technical Report

NREL/TP-5500-74080

June 2019

National Renewable Energy Laboratory 15013 Denver West Parkway Golden, CO 80401

303-275-3000 • www.nrel.gov 


\section{NOTICE}

This work was authored by the National Renewable Energy Laboratory, operated by Alliance for Sustainable Energy, LLC, for the U.S. Department of Energy (DOE) under Contract No. DE-AC36-08GO28308. Funding provided by U.S. Department of Energy Office of Energy Efficiency and Renewable Energy Building Technologies Office. The views expressed herein do not necessarily represent the views of the DOE or the U.S. Government.

This report is available at no cost from the National Renewable Energy Laboratory (NREL) at www.nrel.gov/publications.

U.S. Department of Energy (DOE) reports produced after 1991 and a growing number of pre-1991 documents are available free via www.OSTI.gov.

Cover Photos by Dennis Schroeder: (clockwise, left to right) NREL 51934, NREL 45897, NREL 42160, NREL 45891, NREL 48097, NREL 46526.

NREL prints on paper that contains recycled content. 


\section{Acknowledgments}

The authors would like to acknowledge Amy Jiron, Jordan Hibbs, Cedar Blazek, and the U.S. Department of Energy's Building Technologies Office for supporting this project. This report was prepared by the National Renewable Energy Laboratory (NREL) under Project WBS\# 2.2.2.91, Systems Technology Research and Development.

The authors would also like to acknowledge the significant contributions made by Marta Schantz and Sormeh Konjkav at Waypoint Energy to the study's literature review and market review of connected plug and process load (PPL) meter and control (smart outlet) devices. We thank Bennett Doherty for review of NILM. Additionally, we would like to acknowledge Adam Hirsch with Torque Interactive Media for his editorial contribution and review.

This work was authored by Alliance for Sustainable Energy, LLC, the manager and operator of the National Renewable Energy Laboratory for the U.S. Department of Energy (DOE) under Contract No. DE-AC36-08GO28308. Funding provided by U.S. Department of Energy Office of Energy Efficiency and Renewable Energy Building Technologies Office. The views expressed in the article do not necessarily represent the views of the DOE or the U.S. Government. The U.S. Government retains and the publisher, by accepting the article for publication, acknowledges that the U.S. Government retains a nonexclusive, paid-up, irrevocable, worldwide license to publish or reproduce the published form of this work, or allow others to do so, for U.S. Government purposes. 


\section{List of Acronyms}

AFDD

BAS

DOE

DR

EMIS

FDD

HVAC

IT

LBNL

NILM

NREL

PPL automated fault detection and diagnostics

building automation system

U.S. Department of Energy

demand response

energy management information system

fault detection and diagnostics

heating, ventilating, and air conditioning information technology

Lawrence Berkeley National Laboratory

nonintrusive load monitoring

National Renewable Energy Laboratory

plug and process loads 


\section{Executive Summary}

Plug and process loads (PPLs) already represent a significant fraction of total energy use in commercial buildings and their contribution is expected to increase in the future. Although plugin devices are typically not monitored or controlled, new wireless technologies are being developed that can provide these functions. These "smart" outlets collect data from connected devices and transmit it to cloud services for energy measurement, tracking, and control purposes.

In parallel, energy management information system (EMIS) platforms are being developed and sold to collect and visualize building data and to control other building systems like lighting and heating, ventilating, and air conditioning. Often these smart building platforms optimize individual building systems rather than the building as a whole. Today, smart PPL systems and EMIS technologies have developed to the point that the stage is set to integrate PPLs, lighting, and heating, ventilating, and air conditioning into EMIS platforms for whole-building management, energy optimization, and delivery of demand response grid services.

Based on a literature review, a recent industry roundtable, and a U.S. Department of Energysponsored technology field validation of an advanced wireless smart PPL meter and control (smart outlet) system, this paper describes emerging PPL technologies, the characteristics necessary for successful integration into EMIS platforms, and research questions the U.S. Department of Energy and the national laboratories can pursue to rapidly advance the state of the art.

The research conducted in this investigation was guided by the following questions:

- What is the current state of the wireless smart outlet market?

- How are PPL meters and controls already integrating into building automation system or EMIS platforms?

- At present, how easy is integration?

- At present, how costly is integration?

- Where is the market headed?

- What gaps need to be filled to push the market forward?

The research identified ten important characteristics for more easily integrating PPLs into EMIS and building automation system platforms:

- Connectivity and system robustness

- Local and remote access to data

- Streamlined data management

- Streamlined integration of PPL data into EMIS platforms

- Interoperability with other building end-use systems and platforms

- Control automation

- Demand response capabilities

- Automatic and dynamic load detection (for better plug-and-play capabilities)

- Robust cybersecurity practices

- Ease of deployment and scalability. 
Finally, three major areas were identified as the highest near-term priorities for U.S. Department of Energy-supported laboratory and field testing:

- Integrating PPL submeter data into EMIS platforms, including research focused on:

- Laboratory testing of the integration of existing wireless smart outlet technologies with EMIS platforms to prove concepts

- Field testing concepts to prove application in real commercial buildings

- Implementation of fault detection and diagnostics or automated fault detection and diagnostics for PPL data.

- Interoperability of PPL data with other building end-use data and energy management platforms, including research on:

- Data collection specifications and taxonomies

- Integration of submetered data from PPLs and other building end uses for more coordinated and optimized building performance and energy consumption

- Advances in grid-interactive controls such as demand response capabilities.

- Development of more advanced automatic and dynamic load detection and control, including research on:

- Improving operation and maintenance protocols for PPL data collection and management

- Improving methods to automatically and dynamically identify connected devices

- Introducing control automation to the quickly growing volume of connected equipment. 


\section{Table of Contents}

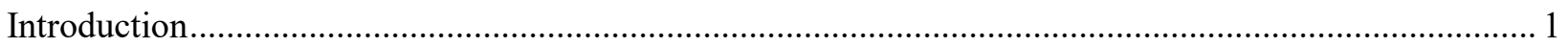

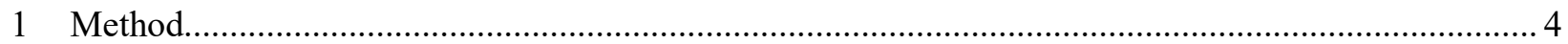

1.1 Survey of Plug and Process Load Control Technologies ........................................................ 4

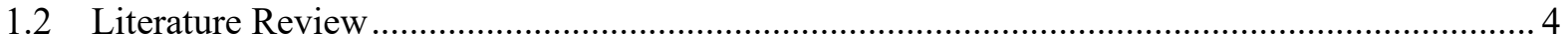

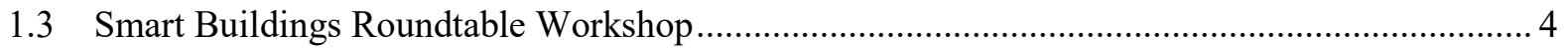

1.4 Wireless Plug and Process Load Smart Outlet Technology Field Validation.............................. 5

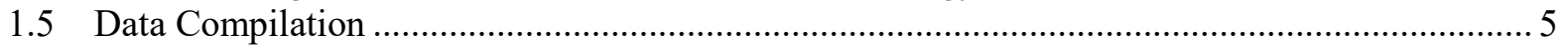

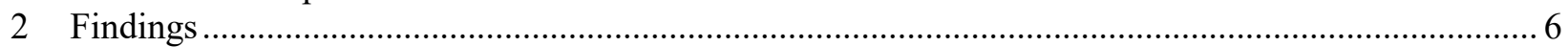

2.1 Goals for Streamlining Connected Plug and Process Load Controls into Building Automation

System and Energy Management Information System Platforms .............................................. 7

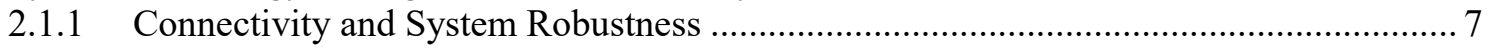

2.1.2 Local and Remote Access to Data....................................................................... 8

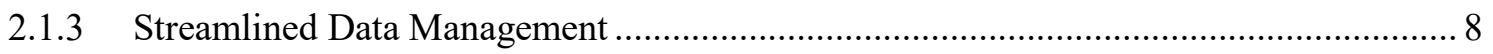

2.1.4 Streamlined Integration of Plug and Process Load Data into Energy Management Information System Platforms ................................................................................ 9

2.1.5 Interoperability with Other Building End-Use Systems and Platforms ......................... 9

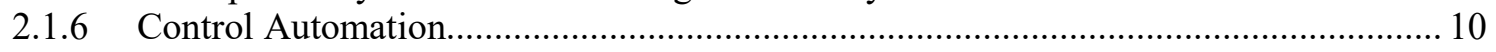

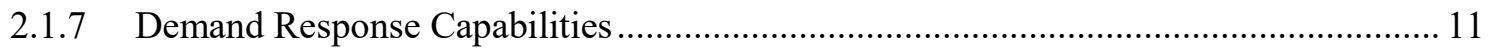

2.1.8 Automatic and Dynamic Load Detection (for Better "Plug-and-Play" Capabilities)..... 11

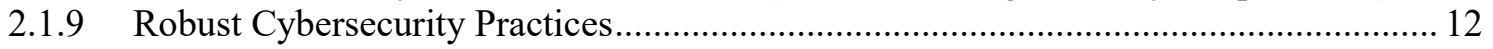

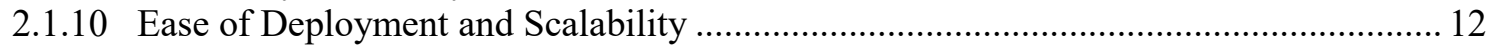

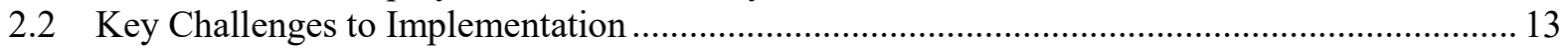

3 Opportunities and Recommendations: Research Needs to Address Challenges ............................... 14

3.1 Integrating Plug and Process Load Data into Energy Management Information System

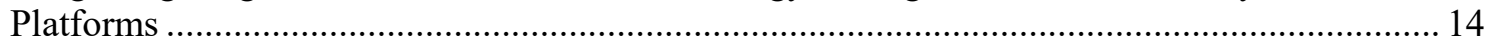

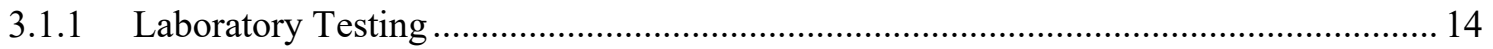

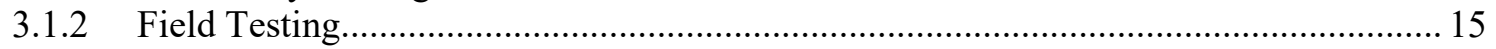

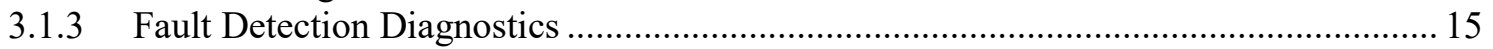

3.2 Interoperability of Plug and Process Load Data with Other Building End-Use Data ................ 16

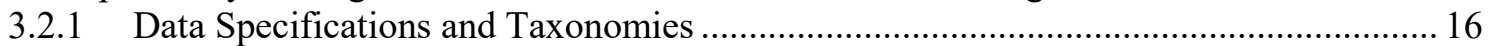

3.2.2 Research on Integrating Submetered Data from Multiple End Uses ............................ 16

3.2.3 Advances in Grid-Interactive Efficient Buildings....................................................... 16

3.3 Development and Testing of Automatic Plug and Process Load Controls ................................ 17

3.3.1 Operational Protocols for Existing Plug and Process Load Meter and Control Technologies (Smart Outlets)................................................................................... 17

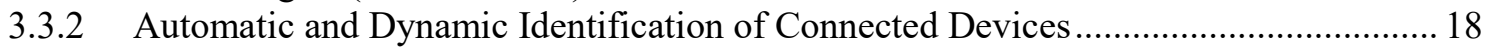

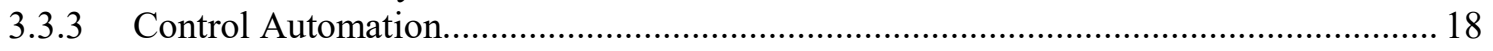

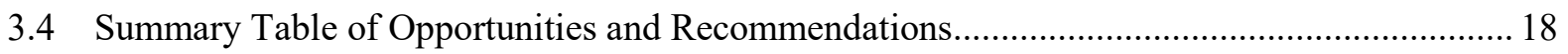

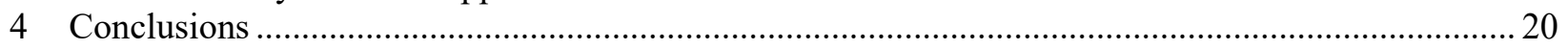

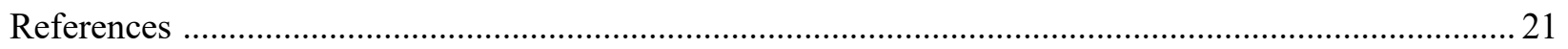

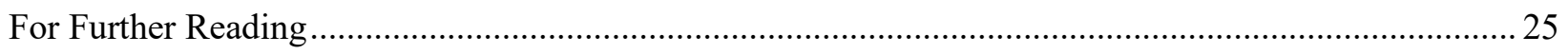

Appendix A: Market Research Summary on Plug and Process Load Technology Solutions that Interface

with Energy Management Information System Platforms ............................................................ 26 


\section{List of Figures}

Figure 1. Percentage of whole-building energy attributed to plug and process loads in 2017 commercial buildings and projections for 2040

\section{List of Tables}

Table 1. Desired Characteristics of Wireless Plug and Process Loads and Smart Outlets ........................ 6

Table 2. Summary of Opportunities and Recommendations ................................................................. 19 


\section{Introduction}

Plug and process loads (PPLs) include all plug-in and hardwired loads in a building that are not associated with heating, ventilating, and air conditioning (HVAC); lighting; water heating; or other major equipment needed for basic building operation. In an office building, for example, plug loads include devices such as computers, monitors, printers, projectors, cell phones, task lights, vending machines, and office kitchen equipment. Process loads include equipment for commercial or industrial processes within a building shell, such as vertical transportation (elevators and escalators) or equipment like industrial washing machines found in large hotels. PPLs comprise a diversity of building end uses, and equipment can vary widely with building type, organizational mission, and individual preference.

PPLs consume a very large portion of whole-building energy use, and they are generally not monitored or controlled, even in buildings that monitor and control other end uses. In 2017, 40\% (7.21 quadrillion Btu) of whole-building energy for U.S. commercial buildings was attributed to PPLs. As Figure 1 shows, this percentage is projected to increase to $49 \%$ (9.21 quadrillion Btu) in 2040 because of increased PPL use and improved efficiency of other building systems such as HVAC, lighting, and building envelope. PPLs can be larger in some building types such as offices, automated warehouses, and call centers. Owners of these types of buildings may be more motivated to implement PPL controls.
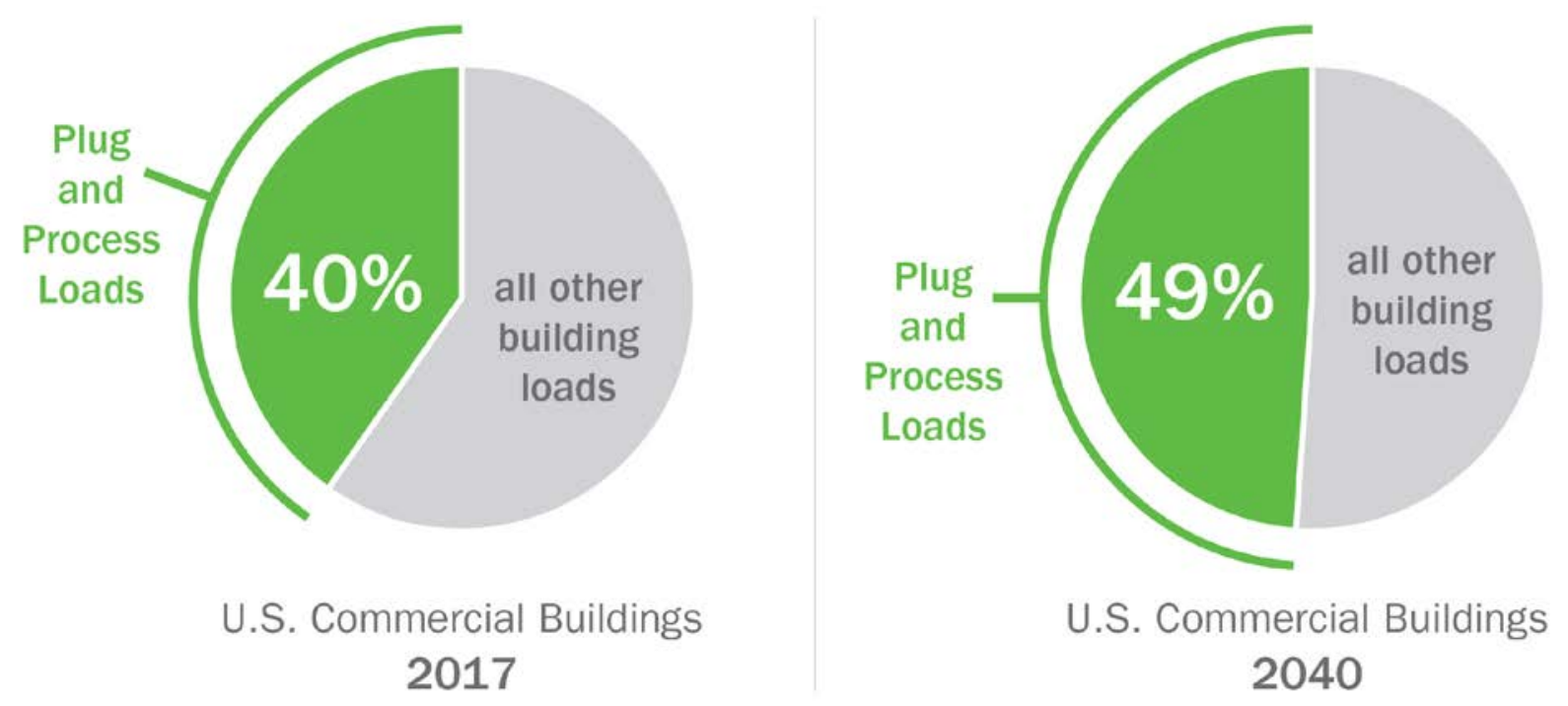

Data source: EIA's Annual Energy Outlook 2018 (EIA 2018)

Figure 1. Percentage of whole-building energy attributed to plug and process loads in 2017 commercial buildings and projections for 2040

Controlling PPLs includes methods and technologies that turn plug-in devices off when not in use. Although it is not common practice to monitor or control PPLs, this capability is becoming more accessible with the emergence of smart, wireless meter and control devices. For electric plug-in equipment, these devices, also called "smart" outlets, enable building owners and managers to measure, track, and control plug-in equipment from proprietary cloud services or through certain building automation system (BAS) platforms. Throughout this paper, the term 
"smart outlet" will often be used to describe smart wireless smart outlet technologies for PPLs. There is also plug-in equipment with the built-in ability to be controlled by a central command. The use cases for these technologies are usually in residential buildings, so that a family can coordinate control through a central hub or application. Examples of these technologies include Google Home, Amazon Alexa, and Apple Home.

Lighting and HVAC systems that use smart, connected meter and control devices to monitor system operation, implement controls, and detect system faults are also becoming more common. However, these smart building systems are typically developed for individual end uses rather than for the building as a whole. This siloed approach limits the use of data management capabilities, data visualization, and equipment controls that can integrate these building end uses together for optimal building performance.

Integrating building end uses and data can be achieved using energy management information system (EMIS) platforms. EMISs represent a broad family of tools and services to manage commercial building energy use. These technologies include energy information systems, equipment-specific fault detection and diagnostic (FDD) systems, benchmarking and utility tracking tools, automated system optimization tools, and BASs (LBNL 2015). EMIS software platforms allow users to view and analyze the various energy use loads that are connected to the system and, in some cases, control those loads.

BAS platforms are designed to control building operations and indoor climate, and typically serve as the foundation of EMIS platforms (LBNL 2015). In the building controls hierarchy, EMIS platforms are overarching and contain BAS platforms that are often "behind the scenes" if they are present. Better integrating PPL controls into BAS and EMIS platforms can yield many energy and nonenergy benefits, including integrated control of multiple building end-use systems for optimal building performance, interoperable data exchange, better data monitoring and analysis, and automated load control. PPL integration into EMIS platforms can also help buildings capture energy savings through operational efficiencies, such as streamlined controls and FDD, as well as cost savings through demand response (DR). Buildings currently respond to DR signals primarily using their HVAC systems and sometimes lighting, so incorporating PPLs would bring the industry closer to a more complete vision for grid-integrated efficient buildings.

The purpose of this landscaping study is to investigate these system integration topics and assess the current market state of wireless meters and controls for commercial PPLs. Specifically, this study focuses mostly on the integration of PPL data into EMIS platforms. Manufacturing process loads are not a major consideration in this study because they are often hardwired with their own controls, and interruptions to their operation could have significant business impacts. The research conducted in this investigation was guided by the following questions: 
- What is the current state of the wireless PPL smart outlet market?

- How are smart outlets already integrating into BAS or EMIS platforms?

- At present, how easy is integration?

- At present, how costly is integration?

- Where is the market headed?

- What gaps need to be filled to push the market forward?

Results from the research were used to identify opportunities to better integrate PPLs into EMIS platforms as well as opportunities for future U.S. Department of Energy (DOE) research. 


\section{Method}

This landscaping study is a summary and analysis of information gained through five pathways. The existing wireless smart outlet market, which offers capabilities to integrate into EMIS and BAS platforms, was investigated through contacts with vendors and internet searches. A literature review of relevant conference papers and peer-reviewed journal articles provided insight into current research in this area. Outcomes from a recent Smart Buildings Roundtable Workshop led by Lawrence Berkeley National Laboratory (LBNL) (Crowe, Kramer, and Granderson 2018) provided expert insight into the usability and effectiveness of these technologies from a building energy management perspective. Additionally, a DOE-led technology field validation on a representative smart outlet product provided perspective from a real-world application (Kandt and Langner 2018). Lastly, insights gained from managing DOE's Better Buildings Alliance Plug and Process Loads Technical Research Team also informed this study's findings (DOE 2018a).

The guiding questions presented in the introduction were used to filter through knowledge gained from these pathways, and findings and opportunities are presented in Sections 2 and 3.

\subsection{Survey of Plug and Process Load Control Technologies}

Current market-ready wireless smart outlet companies that can connect with BAS and EMIS platforms were reviewed. Information on these companies was gathered through conversations with company representatives and internet searches. The companies contacted are listed in Appendix A, with brief summaries of each technology. Some of these technologies transmit information wirelessly through an internet connection or other protocol (like ZigBee), while others are hardwired to the Ethernet. In addition, some of these technologies are structured with a central hub or gateway that collects data from individual sockets.

\subsection{Literature Review}

Approximately 25 accredited journal articles and conference papers (from the past ten years) were selected for review based on their relevancy. These papers cover smart building technologies for PPLs and building control platforms that can integrate PPL data with other building system data. These papers were reviewed with a focus on:

- The current state of the wireless PPL smart outlet market

- The software architecture for integrated controls

- The pros and cons of communication protocols used in each system

- Requirements for integrating PPL data and controls into BAS and EMIS platforms as well as other building systems (i.e., were additional sensor or data flow pathway needs identified?)

- Gaps that need to be filled in order to push the market forward.

\subsection{Smart Buildings Roundtable Workshop}

In February 2018, LBNL invited leading organizations to join other national laboratories and DOE to discuss the current state of smart building systems and their development needs. The meeting's three major objectives were to understand: 
- Commercial building owner motivation to pursue smart building technologies

- The commercial building sector's experience of the usage and uptake of smart building technologies

- The challenges and barriers that building owners face when deploying smart building technologies.

Attendees included energy management team representatives from commercial real estate, higher education, K-12 schools, retail, hospitality, grocery, and government. Outcomes from the roundtable are summarized in a white paper developed by LBNL (Crowe, Kramer, and Granderson 2018). The National Renewable Energy Laboratory (NREL) attended the workshop to strategize and record forward-looking best practices, challenges, and real-world applications of smart building technologies, all of which helped shape the analysis presented in this landscaping study.

\subsection{Wireless Plug and Process Load Smart Outlet Technology Field Validation}

NREL conducted a technology field validation of a representative wireless smart outlet product through DOE's High Impact Technology Catalyst Program (DOE 2018b). The smart outlet company provides a hardware and software solution to manage and control plug-in electric devices in commercial buildings via wireless communication. Smart outlets collect energy data every 15 seconds and communicate them to a gateway via a wireless mesh network. The gateway then sends the data to a cloud-based dashboard, where building owners can review historical data, monitor current values, set up alerts, and implement controls. The system was deployed in two retail stores to verify electricity savings and cost-effectiveness claims as well as to evaluate the system's operability, suitability for different buildings and equipment types, and nonenergy benefits. Findings from this field validation also provided insights for this report. For more information on the specifics of this field validation, refer to Kandt and Langner 2018.

\subsection{Data Compilation}

Work and experiences from the existing technology survey, literature review, smart buildings roundtable workshop, and technology field validation were filtered through the guiding questions. The relevant information was compiled into findings, which are reported in Section 2. Findings include the current state of the market, technology gaps and market needs, and opportunities for future research and development efforts. Section 3 expands on the findings and categorizes research and development opportunities. 


\section{Findings}

The research supporting this landscaping study provided a comprehensive vision for better integrating connected smart outlets into BAS and EMIS platforms. Table 1 summarizes these desirable characteristics, identifies gaps in today's PPL controls market, and lists currently available technology options that can deliver the desired service.

\section{Table 1. Desired Characteristics of Wireless Plug and Process Loads and Smart Outlets}

\begin{tabular}{|c|c|c|}
\hline $\begin{array}{l}\text { Wireless PPL Control } \\
\text { Attributes }\end{array}$ & $\begin{array}{l}\text { Current } \\
\text { Availability Level }\end{array}$ & What is Currently Available? \\
\hline $\begin{array}{l}\text { Connectivity and System } \\
\text { Robustness }\end{array}$ & $\begin{array}{l}\text { Commercially } \\
\text { available }\end{array}$ & $\begin{array}{l}\text { Many connected PPL control platforms currently utilize } \\
\text { unlicensed frequency for operation (such as } \mathrm{Wi}-\mathrm{Fi} \text {, Zigbee, } \\
\text { Bluetooth, etc.). These communication protocols are } \\
\text { resistant to interference, have low data rates, and offer } \\
\text { long battery life. }\end{array}$ \\
\hline $\begin{array}{l}\text { Local and Remote } \\
\text { Access to Data }\end{array}$ & $\begin{array}{l}\text { Commercially } \\
\text { available }\end{array}$ & $\begin{array}{l}\text { Most cloud-based PPL control companies offer local and } \\
\text { remote access to PPL data with user-friendly data } \\
\text { visualization. }\end{array}$ \\
\hline $\begin{array}{l}\text { Streamlined Data } \\
\text { Management }\end{array}$ & $\begin{array}{l}\text { Commercially } \\
\text { available }\end{array}$ & $\begin{array}{l}\text { Connected PPL control platforms often collect and store } \\
\text { granular submetered data. Certain rules can be } \\
\text { implemented to alert users and control outlets; however, } \\
\text { more streamlined approaches are needed to manage } \\
\text { large quantities of PPL data and alerts. }\end{array}$ \\
\hline $\begin{array}{l}\text { Streamlined Integration } \\
\text { of PPL Data into EMIS } \\
\text { Platforms }\end{array}$ & Limited & $\begin{array}{l}\text { Data collected through connected PPL control platforms } \\
\text { can be integrated into BAS and EMIS platforms, but this is } \\
\text { not common practice and more streamlined integration } \\
\text { techniques are needed. }\end{array}$ \\
\hline $\begin{array}{l}\text { Interoperability with } \\
\text { Other Building End-Use } \\
\text { Systems and Platforms }\end{array}$ & Limited & $\begin{array}{l}\text { PPL data can be integrated with certain BAS and EMIS } \\
\text { platforms, but limited resources are available that explain } \\
\text { how PPL data can help inform the control of other building } \\
\text { end uses and vice versa. }\end{array}$ \\
\hline Control Automation & Limited & $\begin{array}{l}\text { PPL controls are currently implemented by a building } \\
\text { energy manager and require constant oversight and } \\
\text { monitoring. Automated controls, such as machine learning } \\
\text { algorithms to determine schedule-based controls, are not } \\
\text { fully developed or on the market yet for PPLs. }\end{array}$ \\
\hline DR Capabilities & Limited & $\begin{array}{l}\text { Some research has been conducted to aggregate PPL } \\
\text { loads and develop load shedding algorithms. More } \\
\text { research is needed to better understand utility needs and } \\
\text { user preferences as well as to integrate PPL controls into } \\
\text { DR capable platforms. }\end{array}$ \\
\hline $\begin{array}{l}\text { Automatic and Dynamic } \\
\text { Load Detection (for } \\
\text { better "plug \& play" } \\
\text { capabilities) }\end{array}$ & Limited & $\begin{array}{l}\text { Radio-frequency identification tags, communicating power } \\
\text { supplies, and nonintrusive load monitoring (NILM) is } \\
\text { available mostly in the residential market. Research is } \\
\text { needed to provide automatic and dynamic load detection } \\
\text { capabilities in the commercial market. }\end{array}$ \\
\hline
\end{tabular}


Table 1. Desired Characteristics of Wireless Plug and Process Loads and Smart Outlets

\begin{tabular}{|c|c|c|}
\hline $\begin{array}{l}\text { Wireless PPL Control } \\
\text { Attributes }\end{array}$ & $\begin{array}{l}\text { Current } \\
\text { Availability Level }\end{array}$ & What is Currently Available? \\
\hline $\begin{array}{l}\text { Robust Cybersecurity } \\
\text { Practices }\end{array}$ & $\begin{array}{l}\text { Commercially } \\
\text { available }\end{array}$ & $\begin{array}{l}\text { Many connected PPL control platforms have some sort of } \\
\text { cybersecurity hygiene in place. However, it is } \\
\text { recommended that facility managers work with information } \\
\text { technology (IT) staff to understand cybersecurity risks, } \\
\text { implement good cybersecurity hygiene, and select } \\
\text { appropriate systems that meet the building needs and } \\
\text { mission. }\end{array}$ \\
\hline $\begin{array}{l}\text { Ease of Deployment and } \\
\text { Scalability }\end{array}$ & $\begin{array}{l}\text { Available, but } \\
\text { immature }\end{array}$ & $\begin{array}{l}\text { Connected PPL control systems are usually simple to set } \\
\text { up, operate, and access data. However, better plug and } \\
\text { play capabilities, and data and alert management could } \\
\text { benefit the industry. Additionally, protocols for managing } \\
\text { cybersecurity risks (for both manufacturer and building } \\
\text { owners) would be beneficial. }\end{array}$ \\
\hline
\end{tabular}

Table 1 demonstrates that the current PPL controls market provides users with good local and remote access to PPL data, data management using data visualization, and some control capabilities. Most systems are also designed to protect user data using modern cybersecurity practices. Generally, operation of these systems is smooth, and the systems are fairly easy to deploy and set up. However, there is room for improvement in several areas before PPL controls can scale, including automatic and dynamic load detection, broad interoperability, control automation, and DR capabilities.

\subsection{Goals for Streamlining Connected Plug and Process Load Controls into Building Automation System and Energy Management Information System Platforms}

Wireless PPL meters and controls are beginning to feature capabilities that support the integration of submetered PPL data into BAS platforms, but these metering and control capabilities are still siloed from the metering and control of other building end-use systems. This "siloing" results in duplicative efforts associated with maintaining separate systems, which increases labor and computing. EMIS platforms could provide the opportunity to integrate PPL data with other building systems such as lighting and HVAC, but more research is needed to do so. This section discusses each of the ten characteristics included in Table 1 and sets a vision for streamlining the integration of PPL data into EMIS platforms. It also covers the current status of those capabilities and what existing technologies - that may be underutilized today - could be leveraged to move development forward.

\subsubsection{Connectivity and System Robustness}

Ease and reliability of communication protocols for PPL monitoring and control systems are fundamental considerations for EMIS and BAS integration. There are many communication protocols on the market that offer different levels of system robustness, connection security, and technological maturity. Some systems will be easier to install, more secure, and more robust than others, and some may become obsolete relatively quickly. It is important to work directly with IT 
staff to determine the best system options for each business, its mission, and the operational time frame.

To provide reliable service, smart PPL control system communications must be resistant to interference (Katipamula et al. 2012). Communications must also be scalable, meaning that the system (and management of system data) does not get overwhelmed as the number of interconnected devices increases dramatically; systems should continue to upload and download data quickly despite an increasing number of devices. Furthermore, the system itself must be robust, not just its communications. Several strategies can help address this concern, including communication protocols that encourage low data rates and long battery life (Katipamula et al. 2012).

Although a great deal of work has been done to improve connectivity and system robustness already, the landscape is always evolving and there is much room for improvement. Wellthought-out, standardized data taxonomies could improve data management, system efficiency, and overall system robustness, enabling better communication pathways between individual devices, their centralized "hubs," and data servers. This would also better support frequent measurements and large amounts of data transfer as more and more devices come online.

\subsubsection{Local and Remote Access to Data}

Most cloud-based PPL monitoring and control companies offer local and remote access to PPL data via user-friendly data visualization applications, making communication with individual monitoring devices easy and convenient (Katipamula et al. 2012; Pipattanasomporn et al. 2015). These technologies typically include smart outlet hardware that collects data and transmits the data wirelessly (or sometimes by wire) to a centralized gateway. The gateway then transfers data through internet protocols to the cloud and users can log into proprietary cloud-based platforms to view the data. Data are typically displayed through various charts and graphs (sometimes customizable) for data visualization. Often, these applications enable the user to aggregate devices or drill down to view and compare the power use of a single device.

\subsubsection{Streamlined Data Management}

EMIS and BAS platforms allow building managers to collect and manage a tremendous amount of data. In order to manage the data today, it is critical to have dedicated personnel to monitor the data streams, look for abnormal energy use patterns, ensure proper operation, implement controls, and maintain the system regularly over time. Good data access and visualization capabilities are a strong point of PPL online dashboards and EMIS platforms, allowing building operators to easily monitor device data and implement controls. As PPL data are integrated into EMIS and BAS platforms, data volume will grow exponentially. As a result, more streamlined data management approaches will be required to collect and store the data and apply algorithms to implement controls or identify when equipment is operating outside of normal bounds.

Alerts that notify building operators that building parameters are out of range must also be monitored by staff who can evaluate the urgency of addressing them. In general, building alerts are triggered by symptoms without insight into the underlying causes, meaning that additional time and expense may go into diagnosing what is really wrong before fixing it; otherwise, actions to address the alert may simply mask the underlying cause, potentially wasting energy 
and allowing the problem to grow worse over time. If the alert is indeed serious, it should be given alarm status, so that buildings operators understand the severity of the malfunction.

Building operators already receive too many alerts, but as more sensors and equipment come online, the number of potential alerts will increase. As a result, a system that can prioritize alerts and maintenance schedules has added value. An EMIS can help prioritize alerts from various systems. Working to ensure that user interfaces provide clear, actionable information will improve these notifications. With clear information, building operators will be able to make faster decisions about how to respond to alerts.

In the future, FDD and automated FDD (AFDD) strategies developed for other building end uses like HVAC could be applied to end-use loads such as lighting and PPLs to provide better system management, identification of faults, alert management, and system integration. Refrigerated plug-in equipment like vending machines and drinking water stations could be ideal candidates for FDD. They have significant per-item loads, so if the power use doesn't match the predicted load, it is easy to detect a fault in the refrigeration system. Other potential vending machine faults include light or light controller failures. Printers could be another plug-in device for FDD consideration.

Additionally, PPL monitoring could complement HVAC AFDD by enabling a more accurate calculation of the building's internal gains. Whole-building energy models can use heat gains from PPLs and other sources such as occupants and lighting to calculate the building's thermal loads. In a whole-building energy model, the predicted HVAC energy use is directly impacted by thermal loads and weather, so accurate calculations of internal gains improve the model. HVAC AFDD systems could use these whole-building models to identify HVAC faults.

\subsubsection{Streamlined Integration of Plug and Process Load Data into Energy Management Information System Platforms}

Integrating management and visualization of PPL data and controls with other building end-use data is a critical step forward in the process of streamlining system operation and optimizing building control. Smart building management platforms typically focus on a specific end use like lighting, HVAC, or PPLs in isolation, meaning that building occupants must purchase and manage multiple individual monitoring and control systems with very similar software and optimization structures, which leads to wasteful overlapping. Integration of data across building systems would ease the burden on building operators and enhance their access to - and use ofoperational data, analyses, and load control/monitoring across HVAC, lighting, and PPL end uses. This integration is a key opportunity for EMIS platforms, but there is a need for the various end-use meters and controls to adhere to common data schemas and protocols.

\subsubsection{Interoperability with Other Building End-Use Systems and Platforms}

Building on the previous section, interoperability of building end-use systems (captured through an EMIS platform) would enable the exchange of data between individual systems for more coordinated end-use control and optimized whole-building performance. This approach is more effective than optimizing individual building systems in isolation, a strategy that can pit systems against each other and limit whole-building savings. System interoperability and integration would help buildings enter a new era of more optimized building efficiency and flexible controls to provide services to the electrical grid such as building-to-grid coordination and the ability to 
respond to DR events. For example, when paired with an occupancy sensor, lighting and plug load controls can be coordinated to turn off when a space or area of a building is unoccupied. This signal could also feed into HVAC controls to ramp down heating and cooling supply to that space or area. Furthermore, these signals could feed into other systems, such as scheduling and security systems, to alert personnel about whether a space is occupied.

\subsubsection{Control Automation}

Today, many smart outlets offer schedule-based control options, the ability to override these controls, and the option to use native equipment controls at the same time (Khamphanchai et al. 2015). However, building owners or managers must look at data and develop specific schedulebased controls for individual pieces of equipment. This effort requires an initial setup and continual maintenance to ensure that controls are being implemented properly and are operating as intended.

There are tremendous opportunities for automating controls for PPLs and coordinating them with controls for other building end uses. In the commercial sector, machine learning algorithms are starting to be developed that identify typical use patterns of certain equipment or groups of equipment. These capabilities are currently more common in the residential sector, and additional research is needed to better understand commercial use cases and refine algorithm development for commercial applications. Residential buildings are smaller and have regular occupants, who often are in communication (like families). Residential occupants make mutual decisions, such as turning off lights when leaving a room. Behaviors of these smaller and more intimate groups can be predicted and controlled by the systems, either automatically or through computer or smart phone applications. Commercial buildings are more challenging because there are more occupants, larger quantities of plug-in equipment and more diversity in occupancy schedules, and more occupant turnover. These complexities present challenges in understanding how to categorize and characterize device classes and locations as well as how to implement controls for multiple occupants and space types.

Machine learning algorithms could leverage occupancy sensor data or control data from other building end-use systems, such as lighting or HVAC, to inform plug-in equipment schedules. Research on data flows between these building end uses (and additional sensors, such as occupancy sensors on smart PPL technologies) is needed to coordinate and optimize these controls.

Additionally, building standards such as ASHRAE Standard 90.1 (Section 8.4.2) and California Title 24 (Part 6 Section 130.5) require a portion of select commercial building spaces to have automatically controlled plug load receptacles (ASHRAE 2016; CEC 2016). The controlled outlets must be permanently marked and built into the wall. Control technologies that can be plugged into an existing outlet and subsequently removed do not meet this code. The code requires service shutoff to be based on a schedule, occupancy sensor, or an automated signal from another control system, and receptacles should allow overrides lasting up to 2 hours. These standards encourage PPL control automation, but do not yet require this to come from a central building control system like an EMIS platform. 


\subsubsection{Demand Response Capabilities}

Some research has been conducted on automated load shedding capabilities for DR events, including the development of algorithms to quantify how much load can be shed at any given time from aggregated plug-in devices to meet DR needs (Keles et al. 2015; Arnold, Sankur, and Auslander 2013b). Many individual plug loads have low power settings and can operate with electricity from a battery. Research could help characterize and categorize plug-in devices to better understand which devices could go into low power mode or be powered by batteries to shed energy consumption when a utility sends a DR signal (Arnold, Sankur, and Auslander 2013b; Guo, Wu, and Long 2013). This supports the inclusion of PPLs as another building asset to help manage energy consumption during DR events. It also presents an opportunity to work with utilities to design PPL controls for load management, meeting both the energy saving goals of a building and the load management goals of a utility.

\subsubsection{Automatic and Dynamic Load Detection (for Better "Plug-and-Play" Capabilities)}

Current smart outlet products require personnel to identify and tag each piece of plug-in equipment in order to facilitate proper communication, data logging, and control through the smart outlet system. Even though smart outlet products have systems in place to do this rapidly, the process is still manual and laborious, adding to the operational and maintenance costs of PPL control systems and potentially introducing errors during human intervention. The laborious process is a barrier that keeps some building owners from adopting the technology because they may not have the time or personnel to set up the system and routinely monitor it. In addition, this approach to building PPL architecture in EMIS or BAS platforms is static, meaning that any reconfiguration can require further work to update the system's data model. Automating this process would significantly streamline interconnection of PPLs, reduce costs, make the system more flexible, and make the resulting EMIS or BAS more reliable. Although they are underutilized, several technologies exist that could improve the situation, including:

- Integrated product identity at manufacturing (Lanzisera et al. 2014) and standardized data architecture and naming if a product contains multiple monitoring points. This can be accomplished by incorporating radio-frequency identification tags into individual plug-in devices at manufacturing, paired with a smart outlet that can read the radio-frequency identification tag. It can also be accomplished by using communicating power supplies (Lanzisera et al. 2014) to dynamically discover when new devices come online or go off-line (Arnold, Sankur, and Auslander 2013a; Pipattanasomporn et al. 2015).

- Internet of things technologies with the ability to communicate with the EMIS or BAS built into the technology. The internet of things device would communicate directly with the EMIS or BAS. Examples from residential application include how Nest thermostats or Phillips hue lightbulbs seamlessly connect to Google Home.

- NILM to disaggregate data and identify specific loads. NILM techniques seek to uncover disaggregated plug load information without the extensive hardware required for smart outlets. Due to its relatively low cost, NILM has been a popular technology in the residential sector dating back to the 1990s; skepticism still exists, however, with regard to its accuracy (Hart 1992; Mayhorn et al. 2016). NILM has yet to be proven as a viable 
technology in the commercial sector, as larger spaces often have a greater quantity and diversity of devices, as well as duplicate instances of the same device, which can be challenging to differentiate (Kalluri et al. 2015; Kamilaris et al. 2014). Nevertheless, research is being conducted to bring NILM to the commercial sector (Kalluri et al. 2015; Kalluri et al. 2016; Kazmi et al. 2014; Rafsanjani and Ahn 2016). Although NILM remains an attractive technology because of its low-cost potential for load disaggregation, at the commercial scale, smart meters remain the most applicable technology as they often provide control functionality in addition to accurate measurements.

\subsubsection{Robust Cybersecurity Practices}

Many communication technologies and protocols exist to protect systems from external access and malicious tampering, but the market is evolving quickly and it can be overwhelming to navigate options. Fortunately, a number of papers describe current communication protocols and their best applications for specific wireless, connected technologies (Kuzlu, Pipattanasomporn, and Rahman 2015; Khamphanchai et al. 2015; Khamphanchai, Saha, and Rathinavel 2014; Parikh, Kanabar, and Sidhu 2010).

Cybersecurity implications exist for any networked building control system, and it is important for facility managers to work closely with IT staff to understand the risks of cybersecurity attacks; determine the best approach for a building's use case and operational mission; and implement appropriate cybersecurity hygiene through sourcing, purchasing practices, and building operations (Crowe, Kramer, and Granderson 2018). Although cybersecurity technologies today provide autonomous surveillance and protection against cybersecurity risks, it is good practice for building owners, managers, or IT staff to continually check data and system performance for abnormalities that may indicate data breaches. Cybersecurity monitoring could become part of an organization's IT standard operating procedures to ensure routine monitoring.

Cybersecurity also includes protecting the privacy of individuals. Organizations that implement PPL monitoring and control devices should also implement good cybersecurity hygiene to minimize and mitigate security breaches and should take care to ensure that individual privacy is maintained to a reasonable extent. Monitoring policies and data accessibility information should be transparent. Individuals should know who has access to the data as well as how devices are controlled.

\subsubsection{Ease of Deployment and Scalability}

Smart outlets usually feature simple setup, operation, and data access. However, these systems often require users to manually identify connected devices, routinely monitor data, and manually implement controls on individual devices or groups of devices. This can be time-intensive and potentially cost-prohibitive for some building or business owners. Automatic and dynamic load detection of plug-in devices could make these systems scalable, reducing the labor hours needed to set up and maintain them. Streamlined data and alert management through EMIS platforms would also reduce the amount of labor required to manage PPLs. Both improvements would increase uptake, therefore benefiting the industry. Additionally, protocols for managing cybersecurity risks (for both manufacturers and building owners) would improve system robustness. 


\subsection{Key Challenges to Implementation}

Several challenges stand in the way of broad uptake of smart PPL controls and their integration into building-wide EMIS and BAS platforms:

- Making the business case. It can be hard for PPL control champions to make the business case for organization-wide adoption of smart outlets because the up-front costs can be considerable and the energy savings of each individual outlet or device are generally small. Even when an owner does invest in smart controllers, there is often concern about the time and level of effort required to manage, track, and maintain the devices over time (Crowe, Kramer, and Granderson 2018). Another concern is interfering with tenant productivity.

- Automatic and dynamic load detection capabilities, integration, and data management. Most smart outlet products are easy to install and set up; however, regular maintenance is needed to identify individual equipment and ensure that the equipment doesn't get removed or plugged into the wrong (nonsmart) socket. Along with the maintenance required to ensure that sockets are set up correctly, it is prudent to have dedicated staff to monitor data over time to check for energy savings opportunities, alerts, or data inconsistency that might indicate suboptimal or malfunctioning equipment operation or a cybersecurity breach. All of these tasks require additional operation and maintenance time and budget. Lastly, submetered PPL (and often lighting) data are not typically integrated into BAS or EMIS platforms, requiring building managers to access multiple platforms to understand building performance. This process can increase time spent to ensure optimal system and whole-building performance.

- Cybersecurity. Cybersecurity concerns are amplified when dealing with hundreds or thousands of connected devices, especially if they are provided by a variety of vendors. There are also concerns about interoperability of systems, obsolescence of systems due to the rapidly changing technology market, and management of firmware and software upgrades over time (Crowe, Kramer, and Granderson 2018).

- Educated and willing workforce. Training is needed to educate building operations and IT staff to operate these increasingly complicated platforms. Even if capable, staff will have to take steps to routinely monitor and maintain these platforms so they are operating optimally. 


\section{Opportunities and Recommendations: Research Needs to Address Challenges}

There are several research opportunities that could improve the integration of PPLs into BAS and EMIS platforms. The synthesis in Section 2 sets the stage for identifying future research topics. This section highlights research areas that are appropriate for DOE and the national laboratories. Much of the needed PPL and EMIS research is directly connected to DOE's core research areas, including cybersecurity, systems integration, AFDD, DR, utility engagement, smart building technology integration, and grid-interactive efficient buildings.

The three highest priority research areas are:

1. Integrating PPL data into EMIS platforms

2. Interoperability of PPL data with other building end-use data

3. Development and testing of automatic PPL controls.

Another area of research is to investigate the anticipated cost and savings associated with integrating PPL data into EMIS platforms. System administrators need information on setup, operation, and maintenance costs; potential energy savings; and payback periods to make decisions about whether system implementation and integration is feasible. This work will become more important as these technologies get closer to being market-ready but is not highlighted as a high priority research area now.

The next sections suggest research questions and research design frameworks for investigating the high priority research areas. Research from DOE and national laboratories is expected to have broad market impact and act as the "glue" needed to coordinate the efforts of multiple stakeholders in these topic areas.

\subsection{Integrating Plug and Process Load Data into Energy Management Information System Platforms}

Prior research has defined conceptually how PPL data and controls can be integrated into EMIS platforms (Zhang et al. 2016; Khamphanchai et al. 2015; Khamphanchai, Saha, and Rathinavel 2014; Pipattanasomporn et al. 2015; Guo, Wu, and Long 2013; Katipamula et al. 2012). The next steps are laboratory and field testing of how PPL data integrate into specific BAS and EMIS platforms. These studies would investigate how these platforms manage data, operation and maintenance protocols for PPL data collection and management, and the potential for incorporating PPL data into FDD and AFDD software. Additionally, this work would improve the understanding of data flows between building end-use systems.

\subsubsection{Laboratory Testing}

Testing the integration of existing wireless smart outlet technologies with EMIS platforms in a laboratory setting would help prove the concept and work out any issues that may arise. Laboratory testing would also provide an opportunity to research how data streams from PPLs and other building end uses can be integrated for more coordinated and optimized building energy control. Furthermore, recommendations or even protocols for more streamlined and 
impactful data collection from building end uses would emerge. Any EMIS that can incorporate varied building end-use data could be used in this research.

\subsubsection{Field Testing}

Field testing is recommended after controlled laboratory testing is complete and is more appropriate for mid- to late-stage technologies. Field testing would verify data flows between PPL and EMIS platforms and help determine ways to better coordinate PPL data and other building end-use data such as lighting and HVAC. One convenient option for field testing would be to partner with a building that is already enrolled in its local utility's existing DR program to explore this interoperability potential.

The following is a list of technical research questions concerning data management and controls that should be addressed to advance PPL integration with EMIS platforms:

- How easy is it to integrate PPL data into these platforms? How can we make this integration better?

- How easy is it to monitor PPL energy use, implement controls, and manage alerts? Is better data visualization and alert management/prioritization needed?

- How can PPL data be integrated with other building system controls for lighting and HVAC to coordinate system operation and minimize/optimize building energy consumption?

- How easy is it to integrate PPL control systems into legacy BAS platforms?

Additional questions about user experience and user friendliness are also important to investigate through field studies. Ultimately, platforms need to work well for users or market acceptance and adoption will be slow. Research questions include:

- What do users want and need as PPL data are integrated into EMIS platforms? What needs will integrating PPL data into EMIS platforms address?

- Can building managers accurately and reliably use the platform? How easy is it to use?

- How satisfied are building managers with the effort and cost to establish and maintain these platforms compared with the energy saving benefits?

\subsubsection{Fault Detection Diagnostics}

Data from EMIS platforms can be used to detect and diagnose building faults, which potentially waste energy. Current work in this area includes development of whole-building AFDD, which uses physics-based models and machine learning algorithms instead of a rule-based approach to FDD (Lin et al. n.d.). The analytics behind AFDD technologies is something that could cross over to other building end-use systems such as PPLs to greatly improve and automate PPL controls. However, a great deal of research is required to identify the best approach for applying AFDD models to PPL data. Research could include how the data from PPLs can provide a better calculation for internal gains in whole-building energy models, which in turn could be used in AFDD for HVAC. Other research could include the power modes of devices such as refrigeration equipment like vending machines and water coolers and how these could be used to develop expected power loads from the devices. Deviations could indicate faults. 


\subsection{Interoperability of Plug and Process Load Data with Other Building End-Use Data}

There is a need for research on the interoperability of PPL submetered data with other building system data and EMIS data management. This includes interoperability and control integration with the broad spectrum of building automation platforms and other networked building end uses. Standardizing data collection specifications and taxonomies could help move the market towards these goals. Below is a summary of actions that can be taken to improve this area.

\subsubsection{Data Specifications and Taxonomies}

These will allow devices and systems to "speak the same language." Research is needed to develop data specifications and taxonomies for connected PPL control devices that would streamline future data collection practices and comply with existing DOE data tools such as the Building Energy Data Exchange Specification (DOE n.d.-a), Standard Energy Efficiency Database (DOE n.d.-b), and Building Performance Database (DOE n.d.-c). Research could also support existing specifications such as Project Haystack ${ }^{1}$ and encourage additional work to strengthen its offerings.

Furthermore, data specifications and taxonomies can be used to categorize and characterize PPLs so that their loads can be addressed by similar control schemes or certain equipment can be prioritized for control. For example, having clear data specifications and taxonomies could streamline the identification and prioritization of specific equipment for temporary shutdown control during a DR event.

\subsubsection{Research on Integrating Submetered Data from Multiple End Uses}

BAS and EMIS compile submetered data from a variety of building end uses. More research is needed to determine the best way to integrate and optimize this information with PPLs for more coordinated and optimized building system control.

\subsubsection{Advances in Grid-Interactive Efficient Buildings}

Current building DR technology can curb HVAC and some lighting loads to meet utility needs. Because the percentage of whole-building energy use for PPLs is expected to increase, there is a large opportunity for research to be conducted to incorporate PPLs into DR programs.

Additionally, there is an opportunity to coordinate the operation of PPLs with lighting and HVAC systems to enhance DR and grid-interaction capabilities. This could include research in:

- Using data taxonomies to categorize PPLs, as discussed in 3.2.1; this categorization can be used to determine which PPLs are ideal for DR

- Aggregating load shedding capabilities for PPLs and coordination of this load shed with other building end uses

- Modeling predictive control algorithms for DR events.

${ }^{1}$ https://project-haystack.org/ 


\subsection{Development and Testing of Automatic Plug and Process Load Controls}

Automating and streamlining the load detection capabilities of plug-in devices would greatly reduce the time required to set up and maintain smart outlet systems. Opportunities to drive the market forward include the following topics.

\subsubsection{Operational Protocols for Existing Plug and Process Load Meter and Control Technologies (Smart Outlets)}

Further research is needed to develop operational protocols for managing and integrating current smart outlet systems. This research would have a qualitative aspect and include investigations of building occupant behavior. Research questions include:

- What happens when sockets disconnect from the network or equipment is moved? Are there protocols to develop or other research areas to pursue that would help manage these devices?

- What are the practices that each stakeholder should utilize to ensure that the building incorporates good cybersecurity hygiene?

- How should override protocols be incorporated into smart outlet operation?

- What are the best ways to educate building occupants on smart outlet operation, including general use and during times of control, such as during a DR event?

Occupants play a major role in a building's efficiency. Every day, even seemingly innocuous occupant behaviors impact electricity use. For example, choosing to charge a cell phone may seem like a negligible load to an individual, but over time and aggregated across occupants, the load becomes significant. The need for guidelines, rules, and controls is even greater for devices like space heaters that use a lot of energy. Therefore, it can be expected that building occupants will need education about smart outlets and plug load management systems. They need to know how an outlet will be controlled, why it's being controlled, and how to override the system if necessary. Research is needed to determine what this guidance should be and how it could be delivered. Research questions include:

- What are best practices for smart outlet and plug load management system procedures so that they are effective in educating building occupants and encouraging desired behaviors?

- What level of control should building occupants have in overriding the plug load system?

- How should building occupants learn to maintain the integrity of plug load control systems and who, if anyone, should be notified if occupants move electric equipment?

- How can data visualization help managers inform occupant education programs?

- How can data visualization provide targeted feedback to the occupant?

For occupants to understand their energy use, they need to be informed through data visualization or by building management. Research could also focus on whether building management staff are informing occupants about their energy use. Associated research questions include: 
- What are best practices that building management or operations and maintenance staff use for disseminating PPL energy-use information?

- How are building management or operations and maintenance staff informing occupants of energy use?

\subsubsection{Automatic and Dynamic Identification of Connected Devices}

Systems that automatically send an alert when connected plug-in devices come on- and off-line exist (Zhang et al. 2016; Khamphanchai et al. 2015; Khamphanchai, Saha, and Rathinavel 2014; Pipattanasomporn et al. 2015), but are more common in the residential space. There is a large opportunity to conduct research to investigate bringing this need from a proven concept to the commercial building market. Work could be done with entities that have already conducted research on these novel solutions to expand the application to a broader spectrum of plug-in devices and building types. This could help streamline data collection and reduce operation, maintenance, and engineering costs.

\subsubsection{Control Automation}

Machine learning algorithms are starting to be developed by industry partners to recognize PPL use patterns and automatically suggest schedule-based controls to facility managers. With these automated capabilities, facility managers would spend much less time reviewing trended PPL data and determining schedule-based controls for individual pieces of equipment. Even though industry is advancing in this space, there are still opportunities for DOE and the national laboratories to work with industry partners to conduct additional research on categorizing and classifying plug-in devices for more optimized and dispersed application of machine learning algorithms for PPLs. Additionally, there are research opportunities to incorporate algorithms for PPL controls with other building end-use data to help coordinate and optimize end-use control and whole-building energy consumption.

\subsection{Summary Table of Opportunities and Recommendations}

Table 2 summarizes the opportunities and recommendations noted in Section 3. This table is provided as a quick reference. 
Table 2. Summary of Opportunities and Recommendations

\begin{tabular}{|c|c|}
\hline High Priority Research Area & Research Focus \\
\hline \multirow[t]{3}{*}{$\begin{array}{l}\text { Integrating PPL Data into EMIS } \\
\text { Platforms }\end{array}$} & $\begin{array}{l}\text { Laboratory testing of the integration of existing wireless smart } \\
\text { outlets with EMIS platforms to prove concepts }\end{array}$ \\
\hline & $\begin{array}{l}\text { Field testing concepts to prove application in real commercial } \\
\text { buildings }\end{array}$ \\
\hline & $\begin{array}{l}\text { Research methods to apply FDD and AFDD algorithms to } \\
\text { PPL data }\end{array}$ \\
\hline \multirow[t]{3}{*}{$\begin{array}{l}\text { Interoperability of PPL Data with } \\
\text { Other Building End-Use Data }\end{array}$} & $\begin{array}{l}\text { Develop specifications and taxonomies for PPL data } \\
\text { collection to enable more streamlined integration of PPL data } \\
\text { with EMIS platforms and data from other building end uses }\end{array}$ \\
\hline & $\begin{array}{l}\text { Research how data streams from PPLs and other building } \\
\text { end uses can be integrated for more coordinated and } \\
\text { optimized building energy control and AFDD }\end{array}$ \\
\hline & $\begin{array}{l}\text { Explore opportunities for enhanced building-to-grid } \\
\text { interaction, bringing PPL and lighting controls into the } \\
\text { equation for load shedding capabilities }\end{array}$ \\
\hline \multirow{3}{*}{$\begin{array}{l}\text { Development and Testing of } \\
\text { Automatic PPL Controls }\end{array}$} & Develop operational protocols for smart outlet technologies \\
\hline & $\begin{array}{l}\text { Work with industry members to develop and test algorithms } \\
\text { to automatically and dynamically identify connected devices } \\
\text { when they come on- and off-line }\end{array}$ \\
\hline & $\begin{array}{l}\text { Work with industry members to develop and test algorithms } \\
\text { to automate PPL control sequences }\end{array}$ \\
\hline
\end{tabular}




\section{Conclusions}

The importance of PPLs in building energy management is increasing as new products proliferate and other building systems become more efficient. Technologies are emerging to measure and control these devices and efforts are underway to integrate them into wholebuilding EMIS platforms that can provide building-wide energy management, optimization, AFDD, and DR delivery capabilities. However, there are still many research questions that need to be answered to fulfill a vision of an EMIS platform that fully integrates PPLs, particularly in terms of systems integration, interoperability, and automatic and dynamic load detection and control. Today, building systems and smart building systems are still typically managed as isolated silos, system configuration is a laborious process, reconfiguration is problematic, and data and alarms must be monitored and analyzed by building operations staff. As smart PPLs in commercial buildings proliferate, much of the system configuration, data analysis, and control will need to be automated. This paper has described the current state of smart PPL technologies, the gaps that need to be filled to better integrate PPLs into EMIS and BAS platforms, and specific laboratory and field research efforts that can close these gaps and rapidly advance the state of the art of fully integrated EMIS systems. In particular, recommendations are provided for high priority research on integrating PPL controls into EMIS platforms and on interoperability and automatic and dynamic load detection. 


\section{References}

Arnold, Daniel B., Michael D. Sankur, and David M. Auslander. 2013a. “An Architecture for Enabling Distributed Plug Load Control for Commercial Building Demand Response." Paper presented at 2013 IEEE PES Innovative Smart Grid Technologies (ISGT), Washington, DC, USA, February 24-27, 2013, 6 pages. http://ieexplore.ieee.org/abstract/document/6497850/.

Arnold, Daniel B., Michael D. Sankur, and David M. Auslander. 2013b. "Optimal Control of Office Plug-Loads for Commercial Building Demand Response." Paper presented at ASME 2013 Dynamic Systems and Control Conference, Palo Alto, California, USA, October 21-23, 2013, 8 pages.

http://proceedings.asmedigitalcollection.asme.org/proceeding.aspx? articleid=1841153.

ASHRAE [American Society of Heating, Refrigerating, and Air-Conditioning Engineers]. 2016. "ASHRAE Standard 90.1: Energy Standards for Buildings Except Low-Rise Residential Buildings." 388 pages.

CEC [California Energy Commission]. 2016. Building Energy Efficiency Standards for Residential and Nonresidential Buildings. 289 pages. https://www.energy.ca.gov/title24/2016standards/.

Crowe, Eliot, Hannah Kramer, and Jessica Granderson. 2018. Summary of Outcomes of 2018 Smart Building Roundtable Workshop. Lawrence Berkeley National Laboratory Report, 16 pages. https://buildings.lbl.gov/sites/default/files/sb-roundtable-summary.pdf.

DOE. 2018a. "Better Buildings Plug \& Process Loads.” Accessed April 27, 2018. https://betterbuildingsinitiative.energy.gov/alliance/technology-solution/plug-process-loads.

DOE. 2018b. "High Impact Technology Catalyst.” Accessed April 27, 2018. https://www.energy.gov/eere/buildings/high-impact-technology-catalyst-0.

DOE. n.d.-a. "Building Energy Data Exchange Specification.” Lawrence Berkeley National Laboratory. Accessed June 2, 2019. https://bedes.lbl.gov.

DOE. n.d.-b. "Standard Energy Efficiency Data (SEED) Platform.” Lawrence Berkeley National Laboratory. Accessed June 3, 2019. https://www.energy.gov/eere/buildings/standard-energyefficiency-data-seed-platform.

DOE. n.d.-c. "Building Performance Database.” Lawrence Berkeley National Laboratory. Accessed June 3, 2019. https://www.energy.gov/eere/buildings/building-performance-databasebpd.

EIA [U.S. Energy Information Administration]. 2018. Annual Energy Outlook 2018. https://www.eia.gov/outlooks/aeo/pdf/AEO2018.pdf.

Guo, Yanyun, Jing Wu, and Chengnian Long. 2013. "Agent-Based Multi-Time-Scale Plug Load Energy Management in Commercial Building." Paper presented at 2013 10th IEEE International 
Conference on Control and Automation (ICCA), Hangzhou, China, June 12-14, 2013, 6 pages. http://ieeexplore.ieee.org/abstract/document/6565198/.

Kandt, Alicen, and Rois Langner. 2019. Plug Load Management Field Study. National Renewable Energy Laboratory Technical Report, 72 pages. https://www.nrel.gov/docs/fy19osti/72028.pdf..

Kamilaris, Andreas, Balaji Kalluri, Sekhar Kondepudi, and Tham Kwok Wai. 2014. "A Literature Survey on Measuring Energy Usage for Miscellaneous Electric Loads in Offices and Commercial Buildings." Renewable and Sustainable Energy Reviews 34 (June): 536-550. https://www.sciencedirect.com/science/article/pii/S1364032114001981.

Kalluri, Balaji, Sekhar Kondepudi, Harn Wei Kua, Tham Kwok Wai, and Andreas Kamilaris. 2015. “OPLD: Towards Improved Non-Intrusive Office Plug Load Disaggregation.” Paper presented at 2015 IEEE International Conference on Building Efficiency and Sustainable Technologies, Singapore, August 31-September 1, 2015, 6 pages. https://ieeexplore.ieee.org/document/7435865.

Kalluri, Balaji, Andreas Kamilaris, Sekhar Kondepudi, Harn Wei Kua, and Tham Kwok Wai. 2016. "Applicability of Using Time Series Subsequences to Study Office Plug Load Appliances." Energy and Buildings, 27 (September): 399-410.

https://doi.org/10.1016/j.enbuild.2016.05.076.

Katipamula, Srinivas, Ronald M. Underhill, James K. Goddard, Danny J. Taasevigen, Mary Ann Piette, Jessica Granderson, Rich E. Brown, Steven Lanzisera, and Teja Kuruganti. 2012. "Smalland Medium-Sized Commercial Building Monitoring and Controls Needs: A Scoping Study." Pacific Northwest National Laboratory Technical Report 22169, 164 pages. http://www.pnnl.gov/main/publications/external/technical_reports/PNNL-22169.pdf.

Kazmi, Aqeel H., Michael J. O'Grady, Declan T. Delaney, Antonio G. Ruzzelli, and Gregory M. P. O’Hare. 2014. “A Review of Wireless-Sensor-Network-Enabled Building Energy Management Systems.” ACM Transactions on Sensor Networks 10, no. 4 (June): 66.1-66.43. http://dx.doi.org/10.1145/2532644.

Keles, Cemal, Abdulkerim Karabiber, Murat Akcina, Asim Kaygusuz, Baris Baykant Alagoz, and Ozan Gul. 2015. "A Smart Building Power Management Concept: Smart Socket Applications with DC Distribution.” Electrical Power and Energy Systems 64 (January): 679688. https://www.sciencedirect.com/science/article/pii/S0142061514005195.

Khamphanchai, Warodom, Abhishek Saha, and Kruthika Rathinavel. 2014. "Conceptual Architecture of Building Energy Management Open Source Software (BEMOSS)." Paper presented at 2014 IEEE PES Innovative Smart Grid Technologies Conference Europe (ISGT Europe), Istanbul, Turkey, October 12-15, 2014, 6 pages. http://ieeexplore.ieee.org/abstract/document/7028784. 
Khamphanchai, Warodom, Manisa Pipattanasomporn, Murat Kuzlu, and Saifur Rahman. 2015. "An Agent-Based Open Source Platform for Building Energy Management." Paper presented at 2015 ICEEE Innovative Smart Grid Technologies - Asia (ISGT ASIA), Bangkok, Thailand, November 3-4, 2015, 6 pages. http://ieeexplore.ieee.org/abstract/document/7387096/.

Kuzlu, Murat, Manisa Pipattanasomporn, and Saifur Rahman. 2015. "Review of Communication Technologies for Smart Homes/Building Applications.” Paper presented at 2015 ICEEE Innovative Smart Grid Technologies - Asia (ISGT ASIA), Bangkok, Thailand, November 3-4, 2015, 6 pages. http://ieeexplore.ieee.org/abstract/document/7437036/.

Lanzisera, Steven, Andrew R. Weber, Anna Liao, Dominic Pajak, and Alan K. Meier. 2014. "Communicating Power Supplies: Bringing the Internet to the Ubiquitous Energy Gateways of Electronic Devices.” IEEE Internet of Things Journal 1, no. 2 (April): 153-160.

http://ieeexplore.ieee.org/abstract/document/6778069/.

LBNL [Lawrence Berkeley National Laboratory]. 2015. A Primer on Organizational Use of Energy Management and Information Systems (EMIS). Better Buildings Solution Center, U.S. Department of Energy Report, 49 pages.

https://betterbuildingssolutioncenter.energy.gov/resources/a-primer-organizational-use-energymanagement-and-information-systems-emis.

Lin, Guanjin, Stephen Frank, Jessica Granderson, Xin Jin, Rupam Singla, and Amanda Farthing. n.d. Prepublication. A Performance Evaluation for Automated Fault Detection and Diagnosis Protocols for Buildings. Lawrence Berkeley National Laboratory and National Renewable Energy Laboratory Report, 29 pages.

Mayhorn, Ebony T., Gregory P. Sullivan, Joseph Petersen, Ryan S. Butner, and Erica M. Johnson. 2016. "Load Disaggregation Technologies: Real World and Laboratory Performance." 2016 ACEEE Summer Study Energy Efficiency in Buildings, 1-13. https://aceee.org/files/proceedings/2016/data/papers/1_675.pdf.

Parikh, Palak P., Mitalkumar G. Kanabar, and Tarlochan S. Sidhu. 2010. "Opportunities and Challenges of Wireless Communication Technologies for Smart Grid Applications.” Paper presented at 2010 IEEE Power and Energy Society General Meeting, Providence, Rhode Island, USA, September 30, 2010, 7 pages. https://ieeexplore.ieee.org/abstract/document/5589988/.

Pipattanasomporn, Manisa, Murat Kuzlu, Warodom Khamphanchai, Abhishek Saha, Kruthika Rathinavel, and Saifur Rahman. 2015. "BEMOSS: An Agent Platform to Facilitate GridInteractive Building Operation with IoT Devices." Paper presented at 2015 IEEE Innovative Smart Grid Technologies - Asia (ISGT ASIA), Bangkok, Thailand, November 3-4, 2015, 6 pages. http://ieeexplore.ieee.org/document/7387018/.

Rafsanjani, Hamed N., and Changbum Ahn. 2016. "Linking Building Energy-Load Variations with Occupants' Energy-Use Behaviors in Commercial Buildings: Non-Intrusive Occupant Load Monitoring (NIOLM)." Procedia Engineering 145 (January): 532-539. https://www.sciencedirect.com/science/article/pii/S1877705816300455. 
Zhang, Xiangyu, Rajendra Adhikari, Manisa Pipattanasomporn, Murat Kuzlu, and Saifur Rahman. 2016. "Deploying IoT Devices to Make Buildings Smart: Performance Evaluation and Deployment Experience." Paper presented at 2016 ICEEE $3^{\text {rd }}$ World Forum on Internet of Things (WF-IoT), Reston, Virginia, USA, December 12-14, 2016, 530-5.

http://ieeexplore.ieee.org/abstract/document/7845464/. 


\section{For Further Reading}

Khan, Aftab Ahmed, Sohail Razzaq, Asadullah Khan, Fatima Khursheed, and Owais. 2015. "HEMSs and Enabled Demand Response in Electricity Market: An Overview." Renewable and Sustainable Reviews 42 (February): 773-785.

http://www.sciencedirect.com/science/article/pii/S1364032114008703.

King, Jennifer, and Christopher Perry. 2017. Smart Buildings: Using Smart Technology to Save Energy in Existing Buildings. American Council for an Energy-Efficient Economy Report A1701, 55 pages. https://aceee.org/sites/default/files/publications/researchreports/a1701.pdf.

Peng, Changhai, and Kun Qian. 2014. "Development and Application of a ZigBee-Based Building Energy Monitoring and Control System." The Scientific World Journal (August): 528410, 13 pages. https://www.hindawi.com/journals/tswj/2014/528410/.

Thongkhao, Yaowaluk and Wanchalerm Pora. 2016. "A Low-Cost Wi-Fi Smart Plug with OnOff and Energy Metering Functions." Paper presented at 2016 13th International Conference on Electrical Engineering/Electronics, Computer, Telecommunications and Information Technology (ECTI-CON), Chiang Mai, Thailand, June 28-July 1, 2016, 5 pages. http://ieeexplore.ieee.org/abstract/document/7561264/.

Weng, Thomas, Bharathan Balaji, Seemanta Dutta, Rahesh Gupta, and Yuvraj Agarwal. 2011. "Managing Plug-Loads for Demand Response within Buildings." BuildSys '11 Proceedings of the Third ACM Workshop on Embedded Sensing Systems for Energy-Efficiency in Buildings.

Seattle, Washington, USA, November 01, 2011, 6 pages.

https://dl.acm.org/citation.cfm?id=2434024.

Zhao, Peng, Therese Peffer, Ram Narayanamurthy, Gabe Fierro, Paul Raftery, Soazig Kaam, and Joyce Kim. 2016. "Getting into the Zone: How the Internet of Things Can Improve Energy Efficiency and Demand Response in a Commercial Building." Proceedings of ACEEE Summer Study, 2016, Pacific Grove, California, USA, August 21-26, 2016, 12 pages.

https://escholarship.org/content/qt5bm711zk/qt5bm711zk.pdf. 


\section{Appendix A: Market Research Summary on Plug and Process Load Technology Solutions that Interface with Energy Management Information System Platforms}

The list below provides a high-level summary of many market-ready PPL technology solutions with the ability to connect to an EMIS or BAS. The companies' PPL technology solution summaries are ordered alphabetically. This is a sampling of the technologies available at the time of this report and not an exhaustive list. NREL and DOE do not endorse or recommend any specific technologies or companies.

\subsection{BEMOSS-Plus}

$\underline{\text { BEMOSS-Plus }}^{2}$ does not provide its own plug load control widgets but instead is a software platform that is installed on a computer. It is designed to work with load control devices from different manufacturers that operate on different communication technologies and data exchange protocols. These include both new commercially available products that operate on Ethernet, Wi$\mathrm{Fi}$, and ZigBee, as well as legacy devices that operate on serial communications using Modbus RTU and BACnet MS/TP protocols. It also supports Ethernet, Serial, Wi-Fi, and ZigBee communication technologies.

\subsection{Bert: Plug Load Management System}

$\underline{B e r t}^{3}$ provides plug-in hardware and in-wall outlet hardware to measure and control plug and hardwired loads up to $277 \mathrm{~V} / 20 \mathrm{~A}$. The Bert devices run on the Wi-Fi network and are controlled either by the Bertbrain 1000 application or by any BACnet/IP system via the Bert Connect BACnet Gateway.

\subsection{Boss: Smart Plugs/Atmosphere}

$\underline{\text { Boss }}^{4}$ provides plug-in outlet controls ("smart plugs"), which communicate through Wi-Fi (IEEE 802.11) to the Boss Atmosphere software cloud EMIS and support an open application program interface for other EMIS integration.

\subsection{Budderfly: Plug Load Controls}

Budderfly $^{5}$ provides a plug-and-play receptacle replacement for existing outlets. The Budderfly Facility Controller (hardware) controls the outlets via a web-based EMIS software using radio frequency (RF) $910 \mathrm{MHz}$ communication technology with power line communication.

\footnotetext{
${ }^{2}$ http://www.bemoss.org

3 https://bertbrain.com/

4 http://bosscontrols.com/solutions/

5 http://www.budderfly.com/
} 


\subsection{Daintree: Controlscope Smart Wireless Control Solution}

Controlscope $^{6}$ does not include its own plug load control widgets, and instead connects other established ZigBee-based plug load control widgets with its software system. Any PPL control technology that communicates via ZigBee can be connected through Controlscope and is aggregated via BACnet to the EMIS.

\subsection{Digi: Xbee Smart Plug ZigBee}

Digi $^{7}$ provides a plug-in outlet control that connects via ZigBee to the Digi ConnectPort X gateways for energy management.

\subsection{Douglas Lighting Controls: Dialog Centralized Lighting Controls System}

Dialog ${ }^{8}$ lighting controls include relay panel-level controls for lighting and plug load circuits. Connects via Ethernet technology and communicates with BACnet to integrate with the EMIS.

\subsection{Enlighted: Plug Load Controller}

Enlighted $^{9}$ provides a hardwired solution to control power at the individual fixture level. The Enlightened Energy Manager uses a web-based interface connected to the sensor network via Ethernet for measurement and control and uses BACnet/IP protocol to integrate the network with the EMIS.

\subsection{Enmetric: Systems}

Enmetric's ${ }^{10}$ hardware powerport allows for outlet-level measurement and control. The plug load management system sends data through the Enmetric wireless web-based bridge hardware to the Enmetric Servers, connected via Ethernet. Enmetric's plug load management software integrates to EMIS via the web using extensive application program interface extensions.

\subsection{Hubbell: H-MOSS Load:Logic Controls}

$\underline{\text { Hubbell }}^{11}$ sells both a wired and a wireless plug load control unit. The wireless unit communicates with the EMIS via Lutron's ClearConnect RF system, which is compatible with other ClearConnect devices.

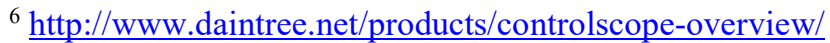

7 https://www.digi.com/products/xbee-rf-solutions/boxed-rf-modems-adapters/xbee-smart-plug-zb

${ }^{8}$ http://www.douglaslightingcontrols.com/products/dialog

${ }^{9} \mathrm{http}: / /$ www.enlightedinc.com/blog/the-enlighted-plug-controller-saves-money-and-makes-buildings-smarter/

${ }^{10}$ https://www.dalchemy.com/

11 http://www.hubbell-wiring.com/press/catalog/E.pdf
} 


\subsection{Ibis Networks: Plug Load Management System}

$\underline{\text { Ibis Networks' }}^{12}$ Plug Load Management System provides a comprehensive plug load solution that starts with plug-in wall sockets ("InteliSockets") that meter and control equipment. The InteliSockets are connected to the InteliGateway via ZigBee and data are reported to a cloudbased InteliNetwork dashboard. The system can also connect to BACnet EMIS systems.

\subsection{Keewi Solutions}

$\underline{\text { Keewi }}^{13}$ is a plug load solution that connects to an outsourced hardware via Z-wave, Zigbee, Retrofit, or in-wall options. Their software uses occupant engagement and plug load analytics strategies with mobile and web apps to communicate to the BAS for granular energy management.

\subsection{Leviton: Receptacle Control Solutions}

$\underline{\text { Leviton }}^{14}$ provides receptacles (outlets) that are controlled by occupancy or schedule-based Leviton devices, which connect wirelessly and have the capability to communicate to LevNet RF. LevNet RF is Leviton's own EMIS solution developed and licensed by EnOcean, although it also has the capability to connect to LON or BACnet through the Leviton Gateway.

\subsection{Osram: WALC (Wireless Area Lighting Controller)}

$\underline{\text { Osram }}^{15}$ provides hardwired controllers to control plug load outlets at the outlet level. It shares data from the GreenBus II enabled devices over ZigBee and connects to the Encelium EMIS via the GreenBus II port BACnet protocol.

\subsection{Tridium: Plug-It}

$\underline{\text { Tridium }}^{16}$ provides plug-in hardware and in-wall outlet hardware to measure and control plug load use. The outlets connect with the JACE software via Wi-Fi and integrate with EMIS using the Tridium Plug-IT communication driver.

\subsection{WattStopper: Lighting Controls and Building Systems}

WattStopper ${ }^{17}$ provides wireless receptacle controls that are controlled using occupancy sensors. The RF-enabled control receptacles communicate wirelessly to the control transmitter and the Infusion Controller II aggregates all WattStopper product information in one system that communicates to EMIS via BACnet.

\footnotetext{
12 http://ibisnetworks.com/ibissystem/

13 http://www.keewisolutions.com/

14 https://www.leviton.com/en

${ }^{15}$ https://www.osram-americas.com/en-us/newsroom/press-releases/Pages/OSRAM-SYLVANIA-AnnouncesWireless-Area-Lighting-Controller-for-ENCELIUM-Energy-Management-System.aspx

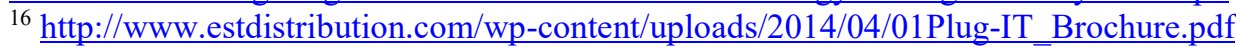

17 http://www.legrand.us/lighting-controls-building-systems.aspx
} 